\title{
Globe
}

Revue internationale d'études québécoises

\section{Bernard Gagnon (dir.), La diversité québécoise en débat. Bouchard, Taylor et les autres, Montréal, Québec Amérique, 2010}

\section{Jocelyn Létourneau}

Volume 14, numéro 1, 2011

URI : https://id.erudit.org/iderudit/1005994ar

DOI : https://doi.org/10.7202/1005994ar

Aller au sommaire du numéro

Éditeur(s)

Globe, Revue internationale d'études québécoises

ISSN

1481-5869 (imprimé)

1923-8231 (numérique)

Découvrir la revue

Citer ce compte rendu

Létourneau, J. (2011). Compte rendu de [Bernard Gagnon (dir.), La diversité québécoise en débat. Bouchard, Taylor et les autres, Montréal, Québec

Amérique, 2010]. Globe, 14(1), 215-219. https://doi.org/10.7202/1005994ar d'utilisation que vous pouvez consulter en ligne.

https://apropos.erudit.org/fr/usagers/politique-dutilisation/ 


\title{
RECENSIONS
}

\author{
Frédéric Rondeau (éd.)
}

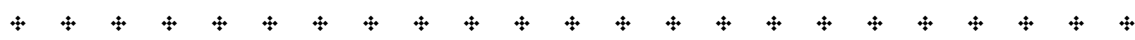

\section{Bernard Gagnon (dir.)}

La diversité québécoise en débat. Bouchard, Taylor

et les autres, Montréal, Québec Amérique, 2010.

Louvrage n'est pas inintéressant. Ceux qui cherchent des perspectives nouvelles sur la question du vivre-ensemble au sein de la société québécoise seront toutefois déçus. Seul le texte d'Alain G. Gagnon, qui fait office de conclusion au livre, apporte quelque argument attrayant pour avancer dans la réflexion sur l'aménagement de la diversité et la promotion de la cohésion sociale au Québec. Réaliste, Gagnon est également nuancé dans ses visions du fédéralisme canadien et pragmatiste dans son appréciation de l'interculturalisme comme paradigme d'intégration sociale. Pour lui, la différence principale entre le multiculturalisme canadien et l'interculturalisme québécois réside dans le fait que le multiculturalisme s'inscrit dans une stratégie de construction "passive» de la citoyenneté alors que l'interculturalisme, plus exigeant et par conséquent plus difficile d'application, cherche à susciter la participation active des membres de la communauté politique à la construction de la société. Pour Gagnon, il y a bien sûr concurrence, dans l'espace politique québécois, entre les deux modèles identitaires, ce qui est fâcheux pour la mise en valeur des possibles inhérents au modèle interculturel québécois. Cette concurrence, toutefois, n'a pas empêché le Québec de se doter d'une espèce de constitution informelle qui offre aux politiques, aux juges, aux acteurs sociaux et à la population en général des points de repère dans la gestion et l'aménagement des interrelations par lesquelles la société québécoise prend forme. Au dire de Gagnon - et cette intuition optimiste semble se concrétiser dans la réalité; on verra si elle se 
confirme dans l'avenir -, «l'implantation du modèle interculturel [québécois] recèle un riche potentiel permettant le rapprochement intercommunautaire en misant sur la confiance [réciproque des citoyens]».

Le point de vue de Gagnon est séduisant parce qu'il évite un certain nombre de biais dans lesquels s'enfoncent plusieurs collaborateurs à l'ouvrage. Parmi ces biais, il y a celui selon lequel l'abîme individualiste et l'anomie sociale marquent décisivement l'évolution actuelle du Québec. Il y a cet autre voulant que la société québécoise souffre d'un problème majeur de cohésion découlant des rapports troubles, voire difficiles, entre une majorité et des minorités. Il y a ce troisième qui veut qu'il soit possible d'organiser ou d'encadrer de manière optimale les relations sociales à l'intérieur d'une société en vue de produire une situation idéale d'intégration collective. Il y a ce quatrième selon lequel l'indépendance politique réglerait une fois pour toutes la question de la gestion de la diversité au Québec.

La faiblesse principale du livre vient de ce qu'aucun auteur (sauf Victor Armony) ne s'est donné la peine de fonder ses prétentions dans des études de terrain ou de se rapporter à des données factuelles. La déconnexion entre la pensée sur le social et la recherche empirique dans la société est en train de devenir le problème numéro un de la sociographie québécoise. On réfléchit beaucoup, mais la réflexion est souvent abstraite, s'abreuvant de grandes théories à forte revendication explicative mais à faible capacité démonstrative. Par exemple, est-il vrai d'affirmer qu'au Québec les identités particulières sont en train de mettre en péril la substance de l'identité collective? Est-il juste d'alléguer que les rapports entre la majorité francophone et les minorités d'autres langues ou cultures s'embrouillent au point de faire problème? Est-il avéré d'arguer que les pratiques de gestion de la diversité sont inefficaces et produisent plus de fragmentation que d'intégration? Percevoir les choses au ras des pâquerettes, en se situant au cœur de la socialité et des sociabilités ambiantes plutôt que sur la passerelle des commentateurs où trônent aussi beaucoup de gérants d'estrade, offrirait sans doute des perspectives différentes, assurément moins pessimistes que celles qui hantent l'esprit de bien des auteurs du recueil.

La deuxième faiblesse du livre tient au manque de perspective ou de profondeur historique des analyses produites. Nombreux sont les auteurs qui postulent que le paradigme de la diversité tel qu'il est pensé au Québec et tel qu'il est assumé par la commission Bouchard-Taylor, c'est-à-dire articulé aux idéaux abstraits et aux valeurs universelles de la modernité, porte mal en lui la continuité d'une expérience historique particulière et d'une tradition nationale spécifique, celle du Québec (français). Derrière cette critique, on 
trouve l'idée selon laquelle il y a une déconnexion entre le projet pluraliste prôné par la commission Bouchard-Taylor et l'historicité du projet éthicopolitique propre au Québec. Dans son article, Joseph Yvon Thériault affirme ainsi que les valeurs mises en avant par la commission Bouchard-Taylor, pour définir les éléments de la culture commune susceptibles de rassembler les membres de la communauté politique québécoise, sont "très peu québécoises». Dit autrement, le devenir québécois est ou serait, dans le paradigme de la diversité promu par la commission Bouchard-Taylor, envisagé en dehors ou au-delà de l'historicité québécoise. Convenue, cette affirmation parait également surfaite. On se demande en effet quels sont les éléments de cette "historicité québécoise particulière " que l'on se plaît à poser sans toujours décrire. De même, en quoi les valeurs rassembleuses identifiées par la commission Bouchard-Taylor sont-elles incompatibles, ou insuffisamment compatibles, avec l'expérience historique québécoise? La Commission cherche-t-elle vraiment à construire le devenir de la société québécoise sur des bases fondamentalement différentes de ce qu'a été le passé de cette société? Peut-être la lecture de l'expérience historique québécoise faite par Bouchard et Taylor est-elle simplement différente de celles que préconisent certains collaborateurs à l'ouvrage. Dans ce cas, il ne s'agit pas d'affirmer péremptoirement que les commissaires ont tort. Il faut encore démontrer que leur compréhension de l'historicité québécoise, dont les contours sont difficiles à cerner et l'" essence» encore moins saisissable, est inadéquate. Or cette démonstration est absente des textes du recueil, qui pâtissent d'un déficit de sensibilité historique tout autant que d'un manque de subtilité historienne.

Sauf Geneviève Nootens, qui appuie finement les perspectives de la Commission, la très grande majorité des auteurs se fait très critique à l'endroit des positions de Bouchard et de Taylor. Les jugements posés, toutefois, sont souvent faciles et vains. Dans certains cas, ils sont rendus à partir d'une vision utopique ou littéraire de l'idéal interculturel alors même que la Commission, mandatée en ce sens par le gouvernement, avait des objectifs pratiques et visait à définir des règles concrètes et opérationnelles de régulation publique. Dans d'autres cas, la critique est formulée à partir d'une position politique (au fond) partisane: puisque la commission Bouchard-Taylor n'a pas suffisamment fustigé le multiculturalisme canadien ni assez admonesté le fédéralisme d'Ottawa, qui sont à la source de bien des problèmes au Québec (sinon à l'origine de tous les ennuis!), ses positions défendues ont manqué l'essentiel. Certes, les auteurs ne sont jamais aussi simplistes dans leurs propos. Après tout, plusieurs d'entre eux comptent parmi les principaux penseurs du Québec. On sent toutefois, derrière bien des analyses, l'idée 
voulant que la Commission ait failli dans ses positions et propositions parce qu'elle n'a pas su poser ni porter, comme elle aurait dû le faire, la question de la culture nationale et celle du projet national québécois.

Cela n'est pas à dire qu'il n'y a pas, outre le texte d'Alain G. Gagnon qui nous a plu, d'autres articles qui apportent de l'eau au moulin de la réflexion. L'hypothèse de Pierre Langeron selon laquelle les dernières années auraient marqué une rupture entre les deux cultures juridiques constitutives de l'identité québécoise, la française et l'anglo-saxonne, au profit de la seconde, mériterait d'être explorée en évitant toutefois de dramatiser la situation. De même, lorsque Guy Laforest écrit que le rapport Bouchard-Taylor représente «un grand effort intellectuel pour amener le Québec du XXI ${ }^{e}$ siècle à trouver une synthèse entre les valeurs universelles de la modernité libérale et démocratique, d'un côté, et le cheminement de son identité particulière, de l'autre», il voit juste. En affirmant que le pragmatisme et la tolérance doivent l'emporter sur l'idéologie républicaine si l'on veut trouver une voie heureuse vers l'avenir en matière de gestion des rapports au religieux, Martin Geoffroy fait aussi preuve de pertinence.

Dans l'ensemble, le livre contient cependant trop de positions prévisibles, d'argumentations connues et de points de vue stéréotypés. L'ouvrage réunit des auteurs qui, malgré leur statut d'intellectuels majeurs, restent conditionnés dans leurs analyses par leur rapport personnel, d'ordre souvent affectif ou expectatif, à la société québécoise. Du côté des plus nombreux se trouvent ceux qui sont habités par la crainte que cette société disparaisse parce qu'elle ne sait s'affirmer ou ne peut le faire à cause de l'Autre qui refuse de la reconnaitre dans son historicité réputée. Pour ces auteurs, la commission Bouchard-Taylor n'a pas su inventer les conditions permettant de lever l'hypothèque du devenir incertain d'une nation investie par les enjeux de la modernité avancée, au premier chef le pluralisme identitaire. Du côté des moins nombreux campent ceux qui, sans nier les problèmes propres à la reproduction d'une petite société, envisagent l'avenir avec sérénité en tablant sur le fait que la diversité vécue au Québec, bien que marquée d'irritants et de tensions, ne pose pas de problème majeur et que la commission Bouchard-Taylor a su énoncer les bases et les cadres d'un compromis honorable entre les défis d'avenir d'une société et ce que fut l'expérience historique de cette société.

À bien des égards, on trouve dans l'ouvrage les relents de questions qui séparent toute la société québécoise lorsqu'elle se penche sur son devenir: jusqu'à quel point s'ouvrir à l'Autre sans se faire hara-kiri dans la relation? Et comment porter un passé sans renoncer à l'avenir? À ces questions, les 
Québécois n’ont jamais donné de réponse unilatérale, radicale ou sectaire. Ils ont toujours choisi de s'autonomiser dans la collaboration et de s'actualiser dans la continuation. C'est dans la tradition des tensions créatrices entre les pôles contraires, élément central de l'historicité québécoise, que les commissaires Bouchard et Taylor ont inscrit leur vision d'avenir du Québec. À défaut d'être convaincante ou enthousiasmante pour les rêveurs, cette position est sans doute sage et sensée pour la société.

Jocelyn Létourneau Université Laval

Chaire de recherche du Canada en histoire et économie politique du Québec contemporain

\section{Michel Biron}

La Conscience du désert, Montréal, Boréal, 2010.

On doit à Michel Biron deux ouvrages d'une importance certaine, tous deux marqués par la sociocritique. Le premier, La modernité belge, était issu du travail de thèse qu'il a fait en Belgique sous la direction de Jacques Dubois. Le deuxième, L'absence du maître, était consacré aux intérêts québécois. Dans les trois œuvres qu'il y étudiait, celles de Saint-Denys Garneau, Jacques Ferron et Réjean Ducharme, Michel Biron démontrait qu'elles «opèrent une rupture profonde avec les discours de leur temps, mais [que] leur geste demeure isolé, impossible à indexer sur une conscience historique ».

Il reste, dans son troisième ouvrage, La conscience du désert, le sociocritique qu'il était dans les ouvrages précédents mais, comme le titre le suggère, un peu moins soucieux de méthode que de lecture littéraire, au sens fort du mot, surtout dans les derniers chapitres. C'est que ce livre, sous-titré "Essais sur la littérature au Québec et ailleurs», est amené par sa forme même, celle d'une collection d'articles divers, français et surtout québécois, à varier les angles d'approche. Mais une question générale les réunit, qui animait déjà les réflexions du livre précédent. Citons:

Le langage de la rupture a quelque chose d'insensé et d'exorbitant pour le poète québécois (ne parlons pas du romancier ou du dramaturge, qui n'auront recours à ce langage que bien après le poète) : il affaiblit la communauté, il divise au lieu de rassembler. Sorti des rangs, le poète moderne est condamné à une solitude qui ne 